\title{
Empirical Study on Consumption Behavior of Female College Students in Network Environment
}

\author{
Bin $\mathrm{Li}^{1, \mathrm{a}^{*}}$, Su Huo ${ }^{2, \mathrm{~b}}$ and Huanyi Chi ${ }^{3, \mathrm{c}}$ \\ ${ }^{1}$ Jilin Agricultural University, No.2888.Xincheng Street, Changchun, China,130118* \\ 2Jilin University, No. 2699.Qianjin Street, Changchun, China, 130013 \\ ${ }^{3}$ Dongbei University of Finance and Economics, No.217.Jianshan Street, Dalian, China, 116025 \\ a105289129@qq.com, b280339877@qq.com, ' $105289129 @ q q . c o m$
}

Keyword: Network Environment; Female College Students; Physical Consumption

\begin{abstract}
From research objective, this is a process where consumers pursue utility maximization for material life and spiritual life. Also this is a goods process where it is through the trade to obtain the price fair and the quality good. As statistics empirical study, it is also another process to realize the construction of personal value, cultural classification, group classification and reproduction of social relations. We have done some survey and analysis, to get the conclusion is that physical consumption is an important part. It has important social and cultural foundations. This paper starts from the perspective of consumer sociology and body sociology. It focuses on the essence and connotation of physical consumption. In the context of current network consumption. Taking female college students as the subject of this study, the article further analyzes and explains the social significance and cultural connotation hidden behind this kind of group consumer behavior.
\end{abstract}

\section{Introduction}

Academically, as one of the important parts of consumption, the female body consumption contains numerous cultural idea and social meanings. In recent years, the consumption of female groups in this area has increased significantly. It has attracted wide attention from all walks of life, which has become one of the hot topics in academic circles. Women, white-collar workers and students are the main parts of the female consumer group. Among these groups, the female college students have received modern teaching, the environmental impact of institutions of higher learning, but the consumption capacity is limited. Therefore, among these groups, they are the most special groups.

However, they have played a significant role in promoting sales in related industries, their spending on physical consumption has increased significantly in recent years. Female college students are an important part of students in colleges and universities. They have modern ideology, active thinking and strong receptivity to new things. They have the cultural literacy and personal qualities matched with education, who are no longer their accessories, have independent personality, have the courage to pursue dreams, have boundless longing for the future, their world is as colorful as the neon at night. With the development of China's economy, people's living standard has been improved. The living expenses of female college students also rise with the provision of their parents, plus their own work-study programs, which make them have stronger purchasing power.

\section{Empirical Design and Hypothesis}

In the research content, mainly expounds and demonstrates the driving factors, current situation and behavior characteristics of their consuming behavior. From the body consumption theory, symbolic theory and social identity theory to interpret and analyze, to bring physical consumption into the sociology of consumption to explore and analyze, thus broaden the scope of the academic, showing the importance and value of the subject of physical consumption. At the same time, this article also deeply explores and analyzes the social concept and cultural connotation hidden behind such consumer behavior. The latest statistics show that women are becoming more of a mainstay of Internet shopping than men. Internet marketing has also always been concerned about female 
customers, from this perspective, this paper mainly studies the physical consumption of female college students in the online consumption environment. The basic condition of female college students' physical consumption was studied by means of questionnaire survey. The main contents of the survey are consumer psychology, consumption impact, specific behavior and other aspects of body consumption. The survey was conducted at an agricultural university in Changchun where the questionnaire was issued 400 copies, and 379 were collected, of which 323 were valid questionnaires, and the effective rate of recovery was $85 \%$.

\section{Survey Results and Analysis}

Facial Consumption. Physical consumption refers to the maintenance of the appearance of a person through a series of consumer activities, Such as make-up, beauty, dress, fitness, maintenance and other consumer activities. Individual body contains static body height, age, dress up and appearance shape, color, health, temperament, image and a series of exterior condition. Individual static appearance dressed up to the body is simply the general situation of the physical consumption, namely, refers to a person of the body, face, to dress up the clothing apparel consumption, hairdressing and cosmetics, etc.

Female college students in school belong to women in the crowd without income groups. Female college students cultural quality is generally high, their view of the world colorful, have their own ideal, have their own life taste, has its own voice, are expected to fully demonstrate their own value, to reach the peak of life. The ideas of female college students are not as conservative as those of traditional women. Their ideas are more open, their modes of thinking are more active, and their receptivity to new things is fast. Although in the economy of female college students can not completely independent, but with China's social and economic development, the domestic living standards improve, they use the family to provide the economic cost of living, as well as his own work-study programs, showed great purchasing power. Survey shows that female college students in consumer spending, cosmetics accounted for $83.5 \%$, monthly expenditure of $0-50$ yuan, accounting for 45\%, 50-100 yuan, accounting for 20.2\%, 100-200 yuan, accounting for $11.7 \%$, 200-400 yuan, accounting for 6.6\%. The group of female college students is the period of youthful beauty, paying special attention to the appearance. According to the survey, $68.4 \%$ of female college students feel that using cosmetics and skin care products can enhance their confidence, their taste and personal charm, Among them, female students account for $27.8 \%$ of the opinions, and only $2.4 \%$ of female students disapprove of this view.

To take female college students samples, Compared the lower grades (freshmen and sophomores) with seniors (juniors and seniors). the lower grade students believe that facial consumption is dispensable, accounting for $26.7 \%$, however The seniors only accounted for $8.8 \%$.Survey shows Senior students also pay more and more attention to facial consumption because of their job hunting, age and other factors, So there's a lot of investment in profile capital.

Physical Consumption. Personal appearance of capital includes face, clothing, accessories, Just as important, it also involves dressing up other parts of the body, People's image and appearance are directly influenced by people's health and body shape. More and more families are paying attention to the role of health care. All kinds of nutrition have become necessary daily consumer goods. In the consumption of health care products, female students with health care accounted for $30.7 \%$.But Consumer health products need a better economic base. In contrast, female students spend less on health care products, Monthly consumption of 1-50 yuan, 50-100 yuan, 100-200 yuan, respectively account for $23.8 \%, 4.9 \%$, and $1.5 \%$. With the rising of living standards, obesity is more and more serious, especially for body shaping is disastrous in order to control obesity levels, to prevent overweight, they begin to choose to lose weight and diet. As a result, women appeared the problem such as anorexia. A variety of diet tea, diet pills also become fashionable for a time. As an auxiliary method, physical exercise has become an important method for women to shape their own shape. The number of consumer sports equipment has increased. For female college students, they have very strict requirements for their body shape, in the young beauty of the period, they have a strong 
desire for beauty, believed to have strong competitiveness cannot do without a good shape, in accordance with the external standard to cognition his self, to make themselves more beautiful slim. According to the survey, female students accounted for $34 \%$ of the body shape. Spend 1-50 yuan, 50-100 yuan and 100-200 yuan on the gym and slimming products, respectively, $24.8 \%, 6.8 \%$, $1.5 \%$.

Self-image capital contains good appearance and slim body, However, there are some differences among the female college students in their pursuit of self image and in the process of seeking social identity and self-identity. Such difference is shown in the freshman and sophomore female students' body consumption idea is relatively low, $9.5 \%$ of the junior female college students don't think keep the beautiful shape and However, the concepts of junior and senior students are higher, because they are looking for jobs, increasing contact with people, and the society has a greater impact on them. Disagree accounted for only $0.4 \%$, in favor of keeping the beautiful shape and appearance is very important for women's life and work of about $98 \%$. By contrast, senior female students found that senior, senior and junior agreed much more.

Clothing Consumption. In the field of consumer sociology, people's clothing and clothing have changed into images and symbols, with some symbolic meanings. We usually use the consumption of external goods to show their worth, and make use of body consumption to obtain social recognition and more self recognition. The survey shows that the areas of urban female consumption continue to expand, and the content of consumption is diversified, but clothing, jewelry consumption has always been in the first place, is the survey of urban women in the consumption of the most. Women spend a lot of money on clothes , and they dress up more and more beautiful. Even among female college students who have no fixed income, the consumption of clothing is also outstanding."My family is not rich. I spend about 400 yuan a month. I don't spend much on food, but I don't have any resistance to clothing consumption, especially for the current epidemic, Every time shopping, buy clothes, pay attention to the style, of course, hope the cheaper the better, put on the new clothes just bought, the mood will be good, full of self-confidence. Due to the condition is limited, the clothes bought are relatively cheap, but they wear clothes together, and the students say they are especially beautiful.

Female college students complete group identification and self-identity through clothing consumption. In reality, the symbol of image symbol clothing mainly has two functions: The first is to recognize the representative meaning of self-image, that is to say, self-image is shaped by clothing, so as to construct a symbol of personalized and high-grade symbolic images, As Craik said, clothing is a "body technology", "through this kind of technology to achieve personal expression and get social point of view". Second is the symbolic meaning of social recognition, clothing is regarded as a symbol, represents a person's identity, content, culture and occupation, etc., is the concentrated expression of interpersonal relations and social relations.

Consumer Behavior Body Tend to Be Networked. In the era of Internet media, online shopping has become the most fashionable expression of consumption tools. The question about "What kind of shopping do you prefer?" Findings, Choose to store the female college students account for $25 \%$ of the proportion of consumption, choose television broadcast shopping accounts for $4.9 \%$, female college students to choose Internet shopping accounts for $32.3 \%$, choose the mobile network such as mobile phone shopping accounts for $37.8 \%$, Thus the network consumption is female college students trend, far more than the traditional shopping way of consumption, that is to say, female college students is the mainstream of network consumption.

Online consumption and female college students' physical consumption are as follows: Firstly, there are many kinds of goods. It can be said that in the network environment, almost all aspects of the product category. Any commodity that the buyer wants can be obtained from it. Because of this, it attracted the majority of female students group of all ages.

Secondly, this shopping takes less time, Compared with what is known as physical stores, such purchases can reduce actual consumption, Buyers through the network to determine the selected goods, network payment after completion, just wait for arrival sign. Such purchases break the constraints of time and space and can easily buy what they need. Therefore, this shopping style has 
been sought after by female students.

Thirdly, with obvious cheap color. Social pressures are surging these days. Make people's life pace compact. Female students are not running in practice every day, or busy studying. They are too busy to take to the streets, and buy the form of cheap, just meet their behavior. Its popularity so that you can imagine. Then, shopping price relative to the entity shop is low, as college students, they are a special existence. There is no direct economic income. Parents provide financial support to them. So, the purchasing power is strongly influenced by price factor. In this case, this kind of shopping form adapted to the consumption characteristics of this group.

\section{Discussion and Conclusion}

As for female college students, they all pay attention to personality. The network media platform can provide the most cutting-edge fashion information. This is more conform to the group definition of beauty and psychological demands. It is undeniable that online shopping is not perfect. Online purchase, unable to soon will be able to get the buy goods. Online shopping, you can not achieve physical selection and try on. After receiving the goods, often there will be "clothes do not fit" phenomenon. Eventually lead to waste of goods, the survey of female college students, we find that their attitudes towards online consumption are mostly supportive. Consumption means network characteristic is the embodiment of body consumption development, Online shopping is more and more popular among female college students in the purchase of clothing, cosmetics, skin care products, health care products and fitness equipment.

Female students have online shopping experience accounted for $83.2 \%$.Accompanied by the promotion of economic strength, the development of body consumption is also reflected in the level of female consumption in the body."When you are online, mainly what kind of goods bought?" The survey found that female college students preferred used in clothing, makeup and other physical consumption accounts for $45 \%$, the second is used in the aspect of cultural consumption such as book, entertainment proportion is $29 \%$, visible female college students' network consumption is given priority to with physical consumption.

\section{Acknowledgement}

This research was financially supported by Jilin province social science fund project: The new media of Jilin rural cultural communication mechanism in the perspective of empowerment (2016B270) and Jilin province education science planning office program: Research on education training mode of communication practical cultivation of talents based on rural culture service(GH170263).

\section{References}

[1] Marx, Engels., Marx and Engels Corpora. People's publishing house,Beijing, 1979.

[2] Geory Simmel. Monetary Philosophy. Beijing: Huaxia publishing Press, Beijing,2002.

[3] Xiu-hua Jiang. Reproductive Behavior of the Female Body Culture Evaluation. Journal of Women's Studies Review, Vol. 66 (2013) No.3, p.25-28.

[4] Li Jing. Gender criticism in slimming advertisements. Journal of xi 'an academy of social sciences, Vol. 83 (2015), No.7, p72-75. 